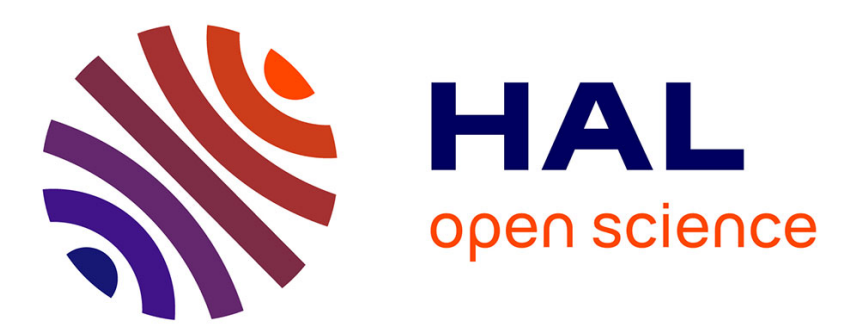

\title{
Dewetting of the residual layer of annealed nanoimprinted polystyrene films
}

Etienne Rognin, Stéfan Landis, Laurent Davoust

\section{To cite this version:}

Etienne Rognin, Stéfan Landis, Laurent Davoust. Dewetting of the residual layer of annealed nanoimprinted polystyrene films. Microelectronic Engineering, 2015, 141, pp.198-202. 10.1016/j.mee.2015.03.063 . hal-01220243

\section{HAL Id: hal-01220243 \\ https://hal.science/hal-01220243}

Submitted on 19 Apr 2020

HAL is a multi-disciplinary open access archive for the deposit and dissemination of scientific research documents, whether they are published or not. The documents may come from teaching and research institutions in France or abroad, or from public or private research centers.
L'archive ouverte pluridisciplinaire HAL, est destinée au dépôt et à la diffusion de documents scientifiques de niveau recherche, publiés ou non, émanant des établissements d'enseignement et de recherche français ou étrangers, des laboratoires publics ou privés. 


\title{
Dewetting of the residual layer of annealed nanoimprinted polystyrene films
}

\author{
E. Rognin ${ }^{\mathrm{a}, \mathrm{b}, *}$, S. Landis ${ }^{\mathrm{a}}$, L. Davoust ${ }^{\mathrm{b}}$ \\ ${ }^{a}$ CEA, LETI, Minatec campus, 17 rue des martyrs, 38054 Grenoble Cedex 9, France \\ ${ }^{\mathrm{b}}$ Grenoble Institute of Technology (Grenoble-INP), CNRS, Univ. Grenoble-Alpes, SIMaP/EPM, 1130 rue de la piscine, BP 75, 38402 Saint-Martin-d'Héres, France
}

\begin{abstract}
The presence of a residual layer at the end of a classical NanoImprint Lithography (NIL) process is at the root of many issues when NIL is used to manufacture resist patterns used later as an etching mask. In this paper, we investigate how the spontaneous break-up of annealed polystyrene films, a phenomenon called dewetting, can produce residual-layer-free polymer patterns. Although the dewetting of flat polymer films has been characterized for decades, little interest has been focused on patterned films. We present exploratory experiments conducted on polystyrene $30 \mathrm{~kg} / \mathrm{mol}$ ultra-thin films. The samples are embossed using thermal NIL with a specific spatially modulated space/line patterned mold, and then annealed at $50^{\circ} \mathrm{C}$ above the glass transition temperature. The resulting patterns are then interpreted as a competition between surface tension and van der Waals forces. Finally, a quantitative analysis is made in the framework of lubrication theory, with the help of a finite volume simulation tool.
\end{abstract}

\section{Introduction}

In a classical thermal NanoImprint Lithography (NIL), a residual layer of polymer is left under the protrusions of the mold [1]. An additional step (usually reactive ion etching) is required to remove the residual layer, jeopardizing the cost-effectiveness and throughput of NIL technology [2]. This aspect is a characteristic difference between NIL and optical lithography where unwanted resist is completely washed away at the development step. On the other hand, ultra-thin polymer films are known to undergo a spontaneous break-up when annealed above their glass transition temperature $\left(T_{g}\right)$, a phenomenon called spinodal dewetting. In particular, the caracteristic time of dewetting depends strongly on the thickness of the film [3]. Especially for films thinner than several nanometers, dewetting can be quite fast, and the way how it may produce residual-layer-free polymer patterns is worth investigating.

The stability of fluid films has been an active field of research regarding fundamental issues, such as intermolecular forces [4,5], as well as applications, such as industrial coating processes [6], thin film patterning [7], or even the study of tear films in the development of contact lens [8]. Extensive reports on dewetting can be

\footnotetext{
* Corresponding author at: CEA, LETI, Minatec campus, 17 rue des martyrs, 38054 Grenoble Cedex 9, France.

E-mail address: etienne.rognin@cea.fr (E. Rognin).
}

found in the reviews by Bonn et al. [9] and Xue and Han [10]. In particular, the dewetting of a polystyrene film on various substrates has been an experimental test case, probably because technologies for coating nanometric polymer films on wafers and characterization means have reached a mature state [11-15]. When a nanometric film is heated above its glass transition temperature, long-range van der Waals forces tend to amplify the thermally induced fluctuations of the free surface. On the other hand, surface tension tends to smooth any protrusion of the fluid film $[16,17]$. As a result of this competition, the film exhibits a characteristic wavelength of instability that depends on the physical parameters of the problem, and finally breaks up into numerous droplets [18].

Most research done in this field has been focusing on flat films and thermally induced fluctuations of the free surface, even though the dynamics of dewetting is largely influenced by the initial shape of the polymer sample as well as of the substrate [22]. For example, a line of fluid on a non-wetting substrate undergoes a PlateauRayleigh instability that breaks the line into droplets. Recent experiments have shown that the resulting droplets can be organized by an initial modulation of the line [23,24]. However, in these particular studies, the residual layer between the lines was first removed by an etching step before any annealing.

In this work, we present dewetting experiments conducted on polystyrene films. Specific spatially-modulated patterns are initially imprinted in the films and a small but continuous residual 
layer is left after the nanoimprint process. Our rather exploratory study aims at understanding the influence of the initial shape of the film on dewetting dynamics. The paper is organized as follows: in the next section we present the experimental method, materials and results. Then two models for the evolution of the film are developed in the framework of lubrication theory: a fully non-linear model for arbitrary film shapes, and a linear model in the case small perturbations of a flat film. Numerical simulations of the non-linear model are reported. We compare and discuss the results in the last section.

\section{Experimental}

\subsection{Method overview}

The method is described on Fig. 1. The polystyrene is spincoated onto a $200-\mathrm{mm}$ silicon substrate (1a). A specific pattern is imprinted in the film using thermal NIL (1b). Demolding is done at room temperature (1c). The sample is annealed on a hot plate at $T_{g}+50{ }^{\circ} \mathrm{C}$ during a definite period of time and then rapidly cooled down (1d). A metallic film (Al-Ti alloy) is created on the polymer film using an evaporation technique (1e). Polystyrene is removed in an ultrasonic bath of toluene (lift-off) (1f).

Both temperature and duration of the annealing (2d) are chosen in order to reach a compromise between the dewetting speed and the accuracy of the temperature steps. Indeed, the higher the temperature above $T_{g}$, the less viscous the polystyrene and the faster the dewetting. On the other hand, the idealized hypothesis of sharp temperature steps is not reasonable for too fast annealings (half a minute in our particular setup [16]), due to thermal transients.

\subsection{Materials and process}

Standard 200-mm ( $\left.8^{\prime \prime}\right)$ mono-crystalline silicon wafers are used. The substrates are cleaned with a $\mathrm{O}_{2} / \mathrm{N}_{2} \mathrm{H}_{2}(2150 \mathrm{sccm} / 240 \mathrm{sccm})$ plasma during $180 \mathrm{~s}$ at $543 \mathrm{~K}$ resulting in the formation of a homogeneous $4 \mathrm{~nm}$-thick $\mathrm{SiO}_{x}$ layer. The molds are made of the same wafers and manufactured at CEA-LETI facilities using

(a)

(b)

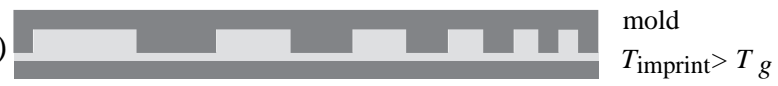

(c)

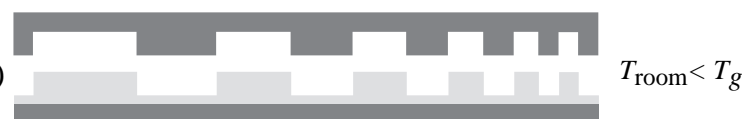

(d)

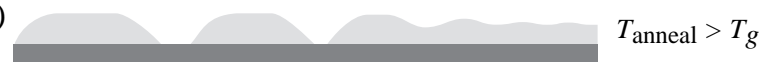

(e)

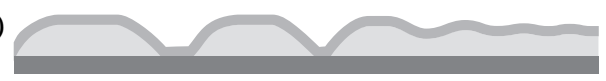

(f)

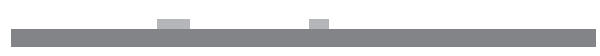

Fig. 1. Schematic cross-section of the experimental method: (a) polymer spincoating. (b) Thermal nanoimprint. (c) Demolding at room temperature. (d) Annealing. (e) Metal deposition. (f) Lift-off. electron-beam lithography and silicon etching processes. The surface of the molds is coated with a fluorinated silane anti-sticking layer. The mold used in this study contains lines of width ranging from $100 \mathrm{~nm}$ to $30 \mu \mathrm{m}$. The pattern is iso-dense, which means that the cumulative width of the protrusions equals the cumulative width of the space between the lines. The depth of the mold is $45 \mathrm{~nm}$.

Low molecular weights $\left(M_{w}=30 \mathrm{~kg} / \mathrm{mol}\right)$ monodisperse $\left(M_{w} / M_{n}<1.06\right)$ polystyrene solution is spin-coated onto the substrates. Any remaining solvent (toluene) is expelled by a quick annealing during $5 \mathrm{~min}$ at $150{ }^{\circ} \mathrm{C}$ in ambient atmosphere. The thickness of the initial film is measured by ellipsometry, and is $50 \pm 1 \mathrm{~nm}$.

The imprints are made with a commercial tool referenced as EVG 520HE from EV Group. The force applied by the screw is $40 \mathrm{kN}$, yielding a pressure of 13 bar on the wafer. Imprints are made at $T_{g}+80^{\circ} \mathrm{C}$ during $10 \mathrm{~min}$. The residual layer is assessed from a combination of ellipsometry near the imprinted pattern and Atomic Force Microscopy (AFM) profiling, and is about $5 \pm 2 \mathrm{~nm}$.

\subsection{Results}

Fig. 2 shows AFM measurements of a nanoimprinted film. After annealing at $150{ }^{\circ} \mathrm{C}$ during $4 \mathrm{~min}$, the area of smaller line widths (right area) is leveled to a large extent, whereas the large line widths (left area) lead to the dewetting of the residual layer.

In order to exhibit the dewetted areas, metal was deposited on the annealed sample, followed by a lift-off in toluene. Optical images of this manufacturing process are collected in Fig. 3. Fig. 3b-d are images of the dewetting phenomenon. In these images, we can see that dewetting occurred for the wider lines (left) as the triple contact lines (edge of the dewetted areas) have a sharp contrast. Contrary to the sample shown in Fig. 2, where the annealing time was $4 \mathrm{~min}$, here, dewetting takes place during $32 \mathrm{~min}$ in order to observe also the long term evolution of the film. We can see strong scratches and Plateau-Rayleigh instabilities [24] which expand over time and influence to a large extent the shape of the final dewetting patterns (Fig. 3d). For smaller lines (right), surface tension overcomes dewetting and smooths the pattern, as we can see by the blurring effect of the annealing.

A Scanning Electron Beam (SEM) image of the metallic patterns is also reported in Fig. 4. From a technological point of view, the defectivity of these lines is high, and this is due to several factors. First, the contact angle of the dewetting polystyrene over the substrate makes it hard to remove the metallic layer near the triple contact line (Fig. 1e), which is likely to produce cracks and defects. Second, the annealing was long enough to trigger line instabilities such as the Plateau-Rayleigh instability mentioned above, so that the straight lines distort and eventually break into droplets. Nevertheless, the purpose of the metallic coating here is not necessarily to manufacture accurate patterns, but it is rather to highlight the dewetted areas.

\section{Theory and simulation}

\subsection{Theory}

Following Oron et al. [18], we model the flow of the film in the framework of lubrication theory. In this theory, the local thickness $h$ of the film is much smaller than the horizontal characteristic length of the film. We assume a no-slip boundary condition between the substrate and the film, and a free surface between the film and the air. The rate of change of $h$ is then governed by the non-linear continuity equation: 


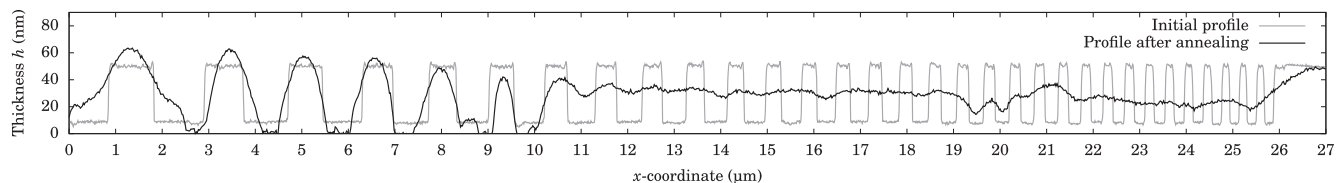

Fig. 2. AFM profiles of a nanoimprinted polystyrene film, before (gray line) and after (black line) annealing during 4 min at $150{ }^{\circ} \mathrm{C}$.

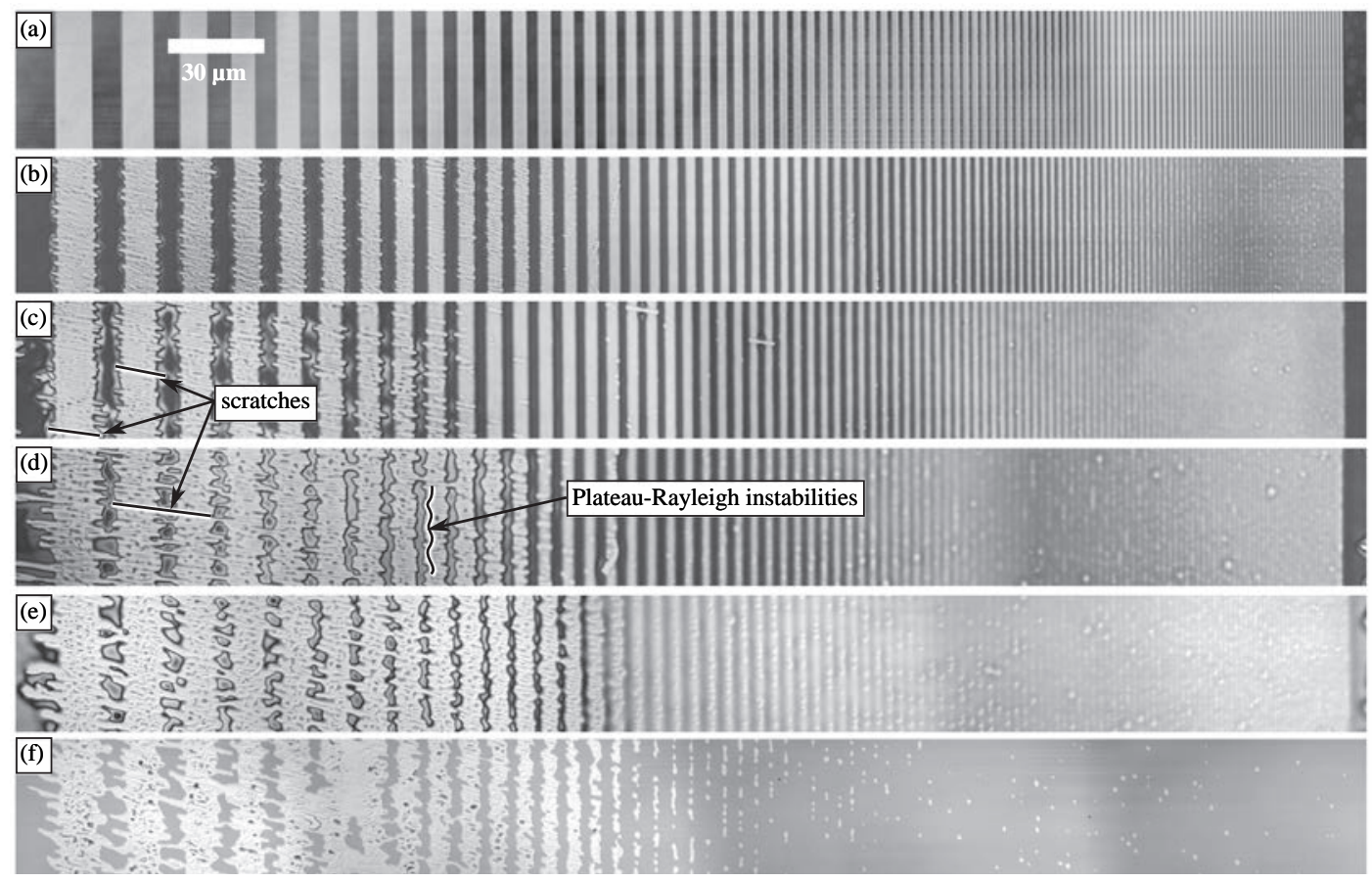

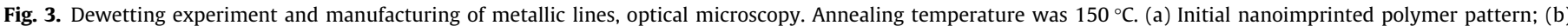

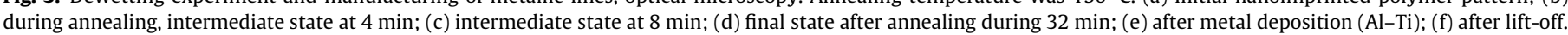

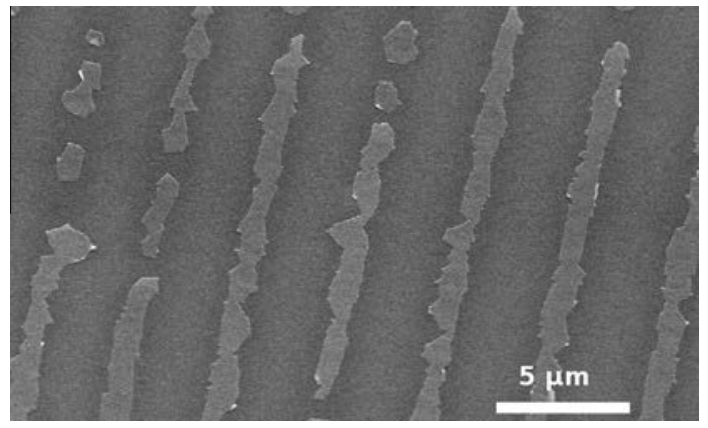

Fig. 4. SEM image of metallic lines after lift-off.

$\frac{\partial h}{\partial t}=\nabla \cdot\left(\frac{h^{3}}{3 \eta} \nabla p\right)$

where $\nabla p$ is the horizontal pressure gradient, and $\eta$ the Newtonian viscosity of the fluid. For nanoscale films, the pressure field is the sum of the Laplace pressure and the disjoining pressure:

$p=-\gamma \kappa+\Pi(h)$,

where $\gamma$ is the surface tension of the free surface, $\kappa$ is the local curvature, and $\Pi$ the disjoining pressure. The disjoining pressure is the effect at the continuum scale of the multiple long-range van der Waals interactions between molecules of both the film and the substrate [19]. The disjoining pressure is the extra pressure developed in the fluid when the thickness of the film is reduced from bulk to ultra-thin film (below about a hundred nanometers for polystyrene).

In lubrication theory, the curvature is approximated by the double derivative (Laplacian) of the thickness: $\kappa=\nabla^{2} h$. In addition, the disjoining pressure is commonly expressed as an Hamaker interaction: $\Pi(h)=-A / 6 \pi h^{3}$, with $A$ the Hamaker constant, so that:

$p=-\gamma \nabla^{2} h-\frac{A}{6 \pi h^{3}}$.

The Hamaker constant $A$ may be positive or negative, depending on the substrate and layers [20]. If $A$ is negative, spinodal dewetting may occur [12].

If we assume that surface tension is homogeneous in the surface, then Eq. (1) can be recast in the dimensionless form:

$\left.\frac{\partial H}{\partial \Theta}=\frac{\partial}{\partial X}-\frac{H^{3}}{(2 \pi)^{4}} \cdot \frac{\partial^{3} H}{\partial X^{3}}+\frac{K}{(2 \pi)^{2} H} \cdot \frac{\partial H}{\partial X}\right)$,

where $H=h / h_{0}$ with $h_{0}$ the average thickness, $X=x / L$ with $L$ the horizontal characteristic length, $\Theta=t / \tau$ with the characteristic time:

$\tau=\frac{3 \eta L^{4}}{(2 \pi)^{4} \gamma h_{0}^{3}}$,

and $K$ is a dimensionless number that quantifies the ratio of the disjoining pressure over surface tension: 
$K=\frac{A L^{2}}{(2 \pi)^{3} \gamma h_{0}^{4}}$.

The $2 \pi$ factors in Eq. (4) are introduced when considering the following perturbative analysis.

\subsection{Linear perturbative analysis}

Let us assume for now that there is no disjoining pressure $(K=0)$, and that the film is flat with a small sinusoidal perturbation. Let $H=1+\varepsilon \cos (2 \pi X)$ with $\varepsilon \ll 1$. Then $H^{3} \approx 1$, and the time evolution for $H$ is, from Eq. (4):

$H(\Theta) \approx 1+\varepsilon \cos (2 \pi X) \exp (-\Theta)$.

Since $\Theta=t / \tau$, the characteristic time $\tau$ is then the relaxation time of a small perturbation of wavelength $L$ [21].

Now, still considering the evolution of a small perturbation, let us add the disjoining pressure $(K \neq 0)$. The thickness evolves as:

$H(\Theta) \approx 1+\varepsilon \cos (2 \pi X) \exp [-\Theta(1+K)]$.

Three cases have to be discussed:

1. $K>0$ : the leveling of the perturbation is accelerated by van der Waals forces. This happens only if the Hamaker constant is positive.

2. $-1<K<0$ : the Hamaker constant is negative but the leveling still occurs, although it is decelerated by van der Waals forces.

3. $K<-1$ : the perturbation grows exponentially at short time (the long-time evolution is governed by the non-linear equation). The instability occurs for a negative Hamaker constant.

If all parameters but $L$ are fixed, the limiting case $K=-1$ corresponds to the critical wavelength $L_{c}$ :

$L_{c}=2 \pi h_{0}^{2} \sqrt{\frac{2 \pi \gamma}{|A|}}$.

\subsection{Finite volume simulations}

Since nanoimprinted films with a thin residual layer present a highly deformed surface, we expect the dynamics of the dewetting to be different from the above linear perturbative analysis. In order to explore and quantify the difference between the linear and nonlinear dynamics, we developed a finite volume simulation tool with the help of FiPy Python library [25]. Eq. (4) is solved for various initial 2D patterns and values of $K$. Boundary conditions at $X=0$ (left boundary of the film) and $X=1$ (right boundary) are set to no flux. An implicit Euler time scheme is used with a time step of $10^{-3}$. Results are shown on Fig. 5 .

Fig. 5a reports the leveling of a small sinusoidal perturbation. The purpose of this case is to test the validity of the code in the linear regime. According to Eq. (7), the decrease of a small perturbation should be proportional to $\exp (-\Theta)$, where $\Theta$ is the dimensionless time. In Fig. 5b, the minimum of the film thickness is plotted over time, along with the theoretical exponential damping. Remarkably, although the amplitude of the perturbation is not so small (20\% of the mean thickness), the pattern follows the exponential leveling predicted by the linear perturbative analysis.

The leveling of a more realistic pattern is illustrated in Fig. 5c. Indeed, the depth of the nanoimprint mold is $45 \mathrm{~nm}$ and we assume a residual layer of $5 \mathrm{~nm}$ (see Section 2). For an iso-dense trench, the average film thickness is then $27.5 \mathrm{~nm}$. In the dimensionless form, the value of the residual layer is then 0.18 . In this simulation, we set the disjoining pressure parameter $K=-0.011$. In thin areas (residual layer), since the no-slip condition is enforced (a)

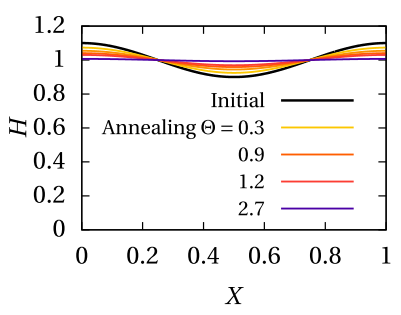

(c)

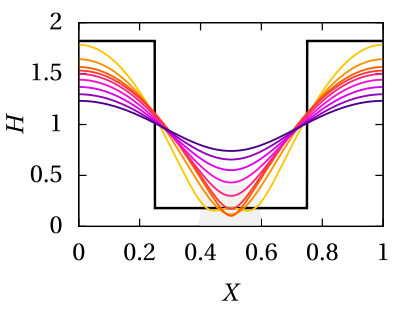

(e)

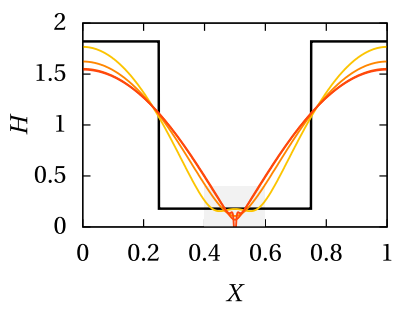

(g) Magnification of (c)

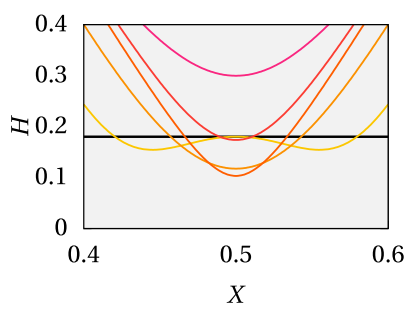

(b)

(d)

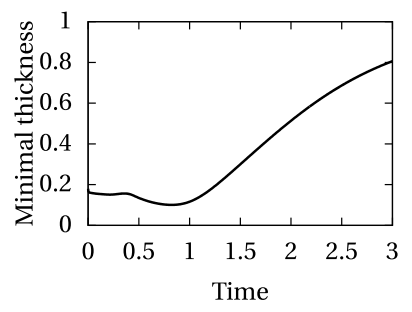

(f)

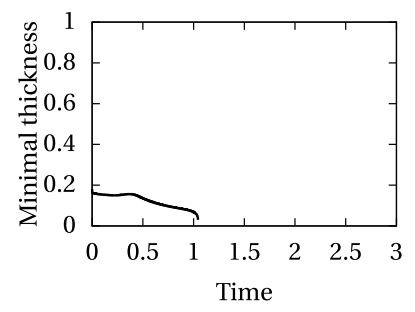

(h) Magnification of (e)

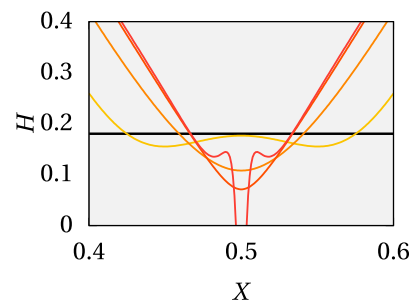

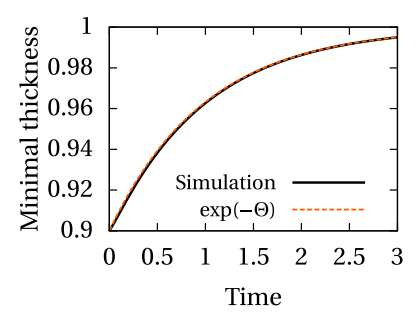

Fig. 5. Simulation of flowing patterns. Left: flowing films. Right: minimum of the thickness over time. ( $a$ and $b$ ) The initial pattern is a small sinusoid, $K=0$ (no van der Waals forces). (c and d) Large amplitude trench, $K=-0.011$. (e and f) Large amplitude trench, $K=-0.012$.

at the bottom of the film, the flow is largely hindered by the substrate, whereas in thicker areas (full thickness) the flow is easier. The resulting effect is that protrusions are deformed and flow faster at the top than at the bottom. Eventually, the pattern recovers a sinusoidal shape. In Fig. 5d, we can see that the minimal thickness does not follow a simple evolution. However, at large time when the amplitude of the pattern is small enough, it follows an exponential decrease, as in the previous case (Fig. 5a and b).

The same initial pattern is used for the simulation reported in Fig. 5e and f. Now the disjoining parameter is set to $K=-0.012$. Around time $\Theta \approx 1$, van der Waals forces take over surface tension and break up the film (Fig. 5f). Here, we see an example of a nonlinear effect. On the one hand, for an infinitely small perturbation of the free surface, $K>-1$ leads necessarily to the leveling of the film. On the other hand, for this particular shape, dewetting occurs even for $K$ as large as -0.012 .

\section{Discussion}

From the dewetting experiments, it should be possible to get at least an order of magnitude of the critical wavelength $L_{c}$ above 
which dewetting occurs. If we consider Fig. 3, this task is not easy because the dewetting limit spreads over a wide range of line widths. In particular in Fig. $3 \mathrm{f}$, continuous metallic lines are gradually replaced by dashes and dots (center of the images). However, the critical length appears more clearly in the AFM profile of Fig. 2. Indeed, dewetting occurred in the left part of the profile for $x<10 \mu \mathrm{m}$, whereas for $x>10 \mu \mathrm{m}$, leveling prevailed. From the design of the mold, we can say that this threshold corresponds to an initial line width of $0.5 \pm 0.1 \mu \mathrm{m}$. We can then estimate $L_{c}=1.0 \pm 0.2 \mu \mathrm{m}$. According to Eq. (6), we can express the Hamaker constant as:

$A=K \frac{(2 \pi)^{3} \gamma h_{0}^{4}}{L^{2}}$.

with $K=-0.012$ from the simulations above, $\gamma=0.032 \mathrm{~N} / \mathrm{m}$ [26], $h_{0}=27.5 \pm 2 \mathrm{~nm}$ and $L=1.0 \pm 0.2 \mu \mathrm{m}$, we obtain: $A=-5 \pm 2 \times 10^{-20} \mathrm{~J}$. This value of the Hamaker constant is in fair agreement with experimental measurements made by Seemann et al. [4] $\left(A=-2.2 \times 10^{-20} \mathrm{~J}\right)$ or computed value by Israelachvili [20] $\left(A=-1.8 \times 10^{-20} \mathrm{~J}\right.$, taking into account the native silicon oxide layer).

On the contrary, if we had used the linearized theory of a fluctuating film, reported as Eq. (9), we would have used $K=-1$, and, since $A$ is proportional to $K$, concluded that $A \sim-5 \times 10^{-18} \mathrm{~J}$, misleading two orders of magnitude. This example illustrates the non-linear nature of film flows when thin residual layers are involved.

\section{Conclusion}

We conducted dewetting experiments on polystyrene ultra-thin films. The films were first imprinted with a specific mold including spatially modulated lines, and then annealed above their glass transition temperature. Unlike the case of a flat film where dewetting is solely triggered by thermally induced fluctuations of the free surface, the films broke up due to the imprinted topography. Dry areas developed in wide trenches whereas the smallest lines were leveled by surface tension. At short time scales, a critical line width separated these two regimes. With the help of numerical simulations, measuring this critical length led to a fair estimate of the Hamaker constant. From a fundamental point of view, this experimental method could be used to study the disjoining pressure in thin films coated on various substrates. On the other hand, work is still necessary to optimize this method for the purpose of removing the residual layer of specific patterns in NIL processes. For example, round shapes such as holes, rings or micro-lenses could be processed with fast annealings in order to avoid any unwanted line instability.

\section{References}

[1] H. Schift, J. Vac. Sci. Technol. B 26 (2008) 458.

[2] S. Landis, Nano Lithography, first ed., Wiley-ISTE, 2010, pp. 87-168.

[3] R. Craster, O. Matar, Rev. Mod. Phys. 81 (2009) 1131.

[4] R. Seemann, S. Herminghaus, K. Jacobs, Phys. Rev. Lett. 86 (2001) 5534-5537.

[5] U. Steiner, J. Polym. Sci. B Polym. Phys. 43 (2005) 3395-3405.

[6] S. Orchard, Appl. Sci. Res. A 11 (1963) 451-464.

[7] R. Seemann, S. Herminghaus, K. Jacobs, J. Phys. Condens. Matter 13 (2001) 4925-4931.

[8] D. Szczesna-Iskander, D. Iskander, Optom. Vis. Sci. 91 (2014) 1406-1411.

[9] D. Bonn, J. Eggers, J. Indekeu, J. Meunier, E. Rolley, Rev. Mod. Phys. 81 (2009) 739.

[10] L. Xue, Y. Han, Prog. Polym. Sci. 36 (2011) 269-293.

[11] C. Redon, F. Brochard-Wyart, F. Rondelez, Phys. Rev. Lett. 66 (1991) 715-718.

[12] G. Reiter, Phys. Rev. Lett. 68 (1992) 75-78.

[13] G. Reiter, A. Sharma, A. Casoli, M.-O. David, R. Khanna, P. Auroy, Langmuir 15 (1999) 2551-2558.

[14] C. Meredith, A. Smith, A. Karim, E. Amis, Macromolecules 33 (2000) $9747-$ 9756.

[15] U. Thiele, Eur. Phys. J. E 12 (2003) 409-416.

[16] E. Rognin, S. Landis, L. Davoust, Phys. Rev. E 84 (2011) 041805.

[17] E. Rognin, S. Landis, L. Davoust, Langmuir 30 (2014) 6963-6969.

[18] A. Oron, S.H. Davis, S.G. Bankoff, Rev. Mod. Phys. 69 (1997) 931-980.

[19] B. Derjaguin, Nature 138 (1936) 330-331.

[20] J.N. Israelachvili, Intermolecular and Surface Forces, second ed., Academic Press, 1991.

[21] T. Leveder, S. Landis, L. Davoust, Appl. Phys. Lett. 92 (2008) 013107.

[22] C. Luo, R. Xing, Y. Han, Surf. Sci. 552 (2004) 139-148.

[23] H. Schift, C. Spreu, A. Schleunitz, J. Lee, Microelectron. Eng. 88 (2011) 87-92.

[24] A. Schleunitz, C. Spreu, J. Lee, H. Schift, J. Vac. Sci. Technol. B 28 (2010) C6M41.

[25] J.E. Guyer, D. Wheeler, J.A. Warren, Comput. Sci. Eng. 11 (2009) 6-15.

[26] J. Bicerano, Prediction of Polymer Properties, CRC Press, 2002. 